\title{
Development and Characterization of Expressed Sequence Tag-Derived Microsatellite Markers for the Wheat Stem Rust Fungus Puccinia graminis f. sp. tritici
}

\author{
S. Zhong, Y. Leng, T. L. Friesen, J. D. Faris, and L. J. Szabo
}

First and second authors: Department of Plant Pathology, North Dakota State University, Fargo 58105; third and fourth authors: U.S. Department of Agriculture-Agricultural Research Service (USDA-ARS) Cereal Crops Research Unit, Northern Crop Science Laboratory, Fargo, ND 58105; and fifth author: USDA-ARS, Cereal Disease Laboratory, University of Minnesota, St. Paul, MN 55108. Accepted for publication 30 October 2008.

\begin{abstract}
Zhong, S., Leng, Y., Friesen, T. L., Faris, J. D., and Szabo, L. J. 2009. Development and characterization of expressed sequence tag-derived microsatellite markers for the wheat stem rust fungus Puccinia graminis f. sp. tritici. Phytopathology 99:282-289.

Puccinia graminis f. sp. tritici is the causal agent of stem rust disease in wheat. The rust fungus has caused devastating disease epidemics throughout history and is still posing a potential threat to wheat production in some regions of the world due to the appearance of new races. To develop microsatellite or simple sequence repeat (SSR) markers for use in population genetics studies, a total of 60,579 expressed sequence tag (EST) sequences (reads) generated from $P$. graminis $\mathrm{f}$. sp. tritici were

screened for tandemly repeated di- and tri-nucleotide units using a bioinformatics approach and 708 unisequences containing putative SSR loci with six or more repeat units were identified. Flanking primers were designed for 384 unique SSR loci, which mapped to different locations of the draft genome sequence of the fungus. Of the 384 primer pairs tested, 72 EST-SSR markers were eventually developed, which showed polymorphism among 19 isolates of $P$. graminis f. sp. tritici and 4 isolates of $P$. graminis f. sp. secalis evaluated. Thirty-two of the SSR loci were also evaluated in three other rust fungi $(P$. triticina, $P$. hordei, and $P$. coronata f. sp. hordei) for cross-species transferability. These SSR markers derived from ESTs will be useful for characterization of population structures and for gene mapping in $P$. graminis.
\end{abstract}

Wheat stem rust, caused by Puccinia graminis f. sp. tritici, has been one of the most devastating diseases in human history. In the early to mid 1950 s, stem rust epidemics caused approximately $50 \%$ yield losses of wheat in North Dakota and Minnesota due to the emergence of a new race (race 15B) $(21,22)$. Since 1974, stem rust has been under control in North America due to the incorporation and pyramiding of stem rust resistance genes into wheat cultivars. The barberry (Berberis vulgaris) eradication program, initiated in the early 1920 s, also maintained the effectiveness of resistant genes longer by reducing the chance of the generation of new virulent races through sexual recombination of the fungus (22). However, wheat stem rust still poses a potential threat to wheat production worldwide. Especially, a new race (Ug99 or TTKS) and its variants were recently found in Eastern Africa and caused serious disease problems in that region $(26,34)$. They have also triggered a serious concern to many other regions of the world where the stem rust resistance genes deployed in commercially grown wheat varieties were not effective against them (13-15).

$P$. graminis f. sp. tritici is a heteroecious macrocyclic fungus with five spore stages (22). The uredia and telia are produced on wheat while the pycnia and aecia are formed on the alternate host, barberry (B. vulgaris). The uredinial stage is the most prevalent stage of the life cycle and can repeat on gramineous hosts under favorable conditions. In the late season, the rust pustules form telia, which produce teliospores. Meiosis occurs in teliospores and basidiospores emerge after teliospores germinate. Basidiospores can infect barberry plants and produce pycnia with pycnio-

Corresponding author: S. Zhong; E-mail address: shaobin.zhong@ndsu.edu

doi:10.1094/PHYTO-99-3-0282

(c) 2009 The American Phytopathological Society spores and receptive hyphae. The receptive hyphae from one mating type can be fertilized by a pycniospore of opposite mating type to produce aecia with dikaryotic aeciospores. The aeciospores infect cereals and reproduce urediniospores again. Significant variation within a $P$. graminis f. sp. tritici population can be generated through genetic recombination during this sexual cycle when barberry plants exist, although several other mechanisms also operate, such as mutation, somatic hybridization, and alien introduction (5). Virulence tests are commonly used to detect pathogen variation and a number of races have been identified. However, virulence markers are limited and subjected to host selection. Since the 1980s, molecular markers have been used to study the genetic variation and population structure of fungi, including $P$. graminis f. sp. tritici. Using enzyme markers combined with virulence evaluation, Burdon and Roelfs (6) compared the sexually and asexually reproducing populations of $P$. graminis f. sp. tritici and found that the sexually reproducing population exhibited higher genetic diversity compared with the asexually reproducing population. Other markers including randomly amplified polymorphic DNA (RAPD) and amplified fragment length polymorphism (AFLP) were also used to analyze genetic diversity in wheat stem rust (24) and other rust fungi $(8,20)$. Although these markers provide powerful tools for population genetic studies, each of them has limitations in application. For the dikaryotic organisms like Puccinia species, codominant markers such as SSRs are more informative in revealing genetic variations when compared to dominant markers (30).

SSR markers have been developed for many species of plants and fungi and are usually developed from genomic DNA through the construction of SSR-enriched libraries. This approach, although effective, is quite time consuming and labor-intensive. In recent years, more and more expressed sequence tags (ESTs) from cDNA libraries have been generated for a number of organisms, 
including fungi. These ESTs have served an important resource for developing new SSR markers to assess genetic diversity. ESTSSRs have been previously developed and characterized from a number of plant species $(9,17,33)$, as well as from fungal species $(10,11)$. In $P$. graminis f. sp. tritici, EST-SSRs were not available although 25 SSR markers were developed from genomic DNA clones of SSR-enriched libraries (32). To develop additional SSR markers, we screened 6,0579 EST sequences of $P$. graminis f. sp. tritici and developed 72 EST-derived SSR markers. These additional SSR markers were characterized using various isolates of $P$. graminis f. sp. tritici and other taxa of rust fungi.

\section{MATERIALS AND METHODS}

Fungal isolates. Nineteen isolates of $P$. graminis f. sp. tritici and four isolates of $P$. graminis f. sp. secalis were used in the study (Table 1). In addition, one isolate from each of the three Puccinia species, i.e., P. triticina, P. hordei, and P. coronata f. sp. hordei, provided by J. Rasmussen, B. Steffenson, and Y. Jin, respectively, was used to test cross-species amplification of the EST-SSR primers developed from $P$. graminis f. sp. tritici. Isolates of $P$. graminis f. sp. tritici were increased on the seedlings of the susceptible variety 'Little Club' (CItr 4066) and those of $P$. graminis f. sp. secalis were increased on the susceptible rye accession 'Prolific' (PI 535177). The seedling plants used for rust increase were treated with malaic hydriate before inoculation to enhance spore production (29). Thirteen of the P. graminis f. sp. tritici isolates were previously characterized for virulence on a set of standard stem rust differentials by Sun and Steffenson (31) and a four letter code was assigned to them based on their infection reactions on the differentials (27). The other $P$. graminis f. sp. tritici isolates were evaluated on the same set of differentials following the same procedure as described by Sun and Steffenson (31).

Generation of EST sequences and identification of microsatellites. Three cDNA libraries were constructed from urediniospores, germinated urediniospores, and teliospores of the $P$. graminis f. sp. tritici isolate 7A (CRL 75-36-700-3), respectively (37). These cDNA libraries were sequenced by the Broad Institute, MIT and 60,579 EST sequences were generated. Assembling of the ESTs into contigs and singlets was performed using the CAP3 program (12). The resulting unique sequences were screened for microsatellite loci with di- and tri-nucleotide combinations tandemly repeated six or more times using a Perl script provided by Zheng Jin Tu at the University of Minnesota, St. Paul. Sequences containing microsatellites were analyzed by BLASTX (1) to identify similarities to previously characterized sequences in GenBank and thereby deduce possible gene function. The BLASTN search was also performed to map these sequences into the supercontigs and contigs assembled from the genomic sequences generated from the isolate 7A of $P$. graminis f. sp. tritici.

Primer design, polymerase chain reaction (PCR) amplification and polymorphism evaluation. Primers were designed within the flanking regions of each microsatellite locus using Primer3 with the default settings: OPT_SIZE $=20$; MIN_SIZE = 18; MAX_SIZE $=27$; MIN_TM $=57$; and MAX_TM $=63$. In order to generate PCR products with fluorescent-labeled M13 primer incorporated, an M13 tag (5'-CACGACGTTGTAAAACGAC) was added to the $5^{\prime}$ end of each forward primer. Primer pairs designed were initially evaluated for amplification using DNA from the isolate QCC-2. PCR was performed in an MJ Research PTC-100 thermal cycler (Watertown, MA) with the following profile: $95^{\circ} \mathrm{C}$ for $5 \mathrm{~min}, 3$ cycles at $95^{\circ} \mathrm{C}$ for $30 \mathrm{~s}, 50^{\circ} \mathrm{C}$ for $30 \mathrm{~s}, 72^{\circ} \mathrm{C}$ for $80 \mathrm{~s}, 33$ cycles at $94^{\circ} \mathrm{C}$ for $15 \mathrm{~s}, 52^{\circ} \mathrm{C}$ for $15 \mathrm{~s}$, and $72^{\circ} \mathrm{C}$ for $45 \mathrm{~s}$, followed by a $4^{\circ} \mathrm{C}$ holding step. Each PCR amplification contained $1 \times$ PCR buffer $(10 \mathrm{~mm}$ of Tris- $\mathrm{HCl}$, $50 \mathrm{~mm}$ of $\mathrm{KCl}$ ), $200 \mu \mathrm{m}$ of dCTP, dGTP, dTTP, and dATP, $1.5 \mathrm{~mm} \mathrm{MgCl}_{2}, 5$ pmol M13 primer labeled by IRD700 or IRD800 (LI-COR, Lincoln, NE) at the 5' end, 1 pmol 5'-tagged forward primer, 5 pmol reverse primer, $1 \mathrm{U}$ of $\mathrm{Taq}$ polymerase (New England Biolabs, Ipswich, MA) and $10 \mathrm{ng}$ of rust genomic DNA in a final volume of $10 \mu \mathrm{l}$. The PCR products were diluted 10- to 20-fold and analyzed on an LI-COR 4300 DNA sequencer (Lincoln, NE) using a 7\% polyacrylamide gel. Primers that gave clear amplicons were further evaluated on 19 isolates of $P$. graminis f. sp. tritici and 4 isolates of $P$. graminis f. sp. secalis for polymorphism analysis. SSR primers shown to be polymorphic among the $P$. graminis isolates were also evaluated on one isolate of $P$. hordei, $P$. triticina and $P$. cronata f. sp. hordei to test the

TABLE 1. Rust isolates used for characterization of microsatellite markers developed from Puccinia graminis f. sp. tritici expressed sequence tags

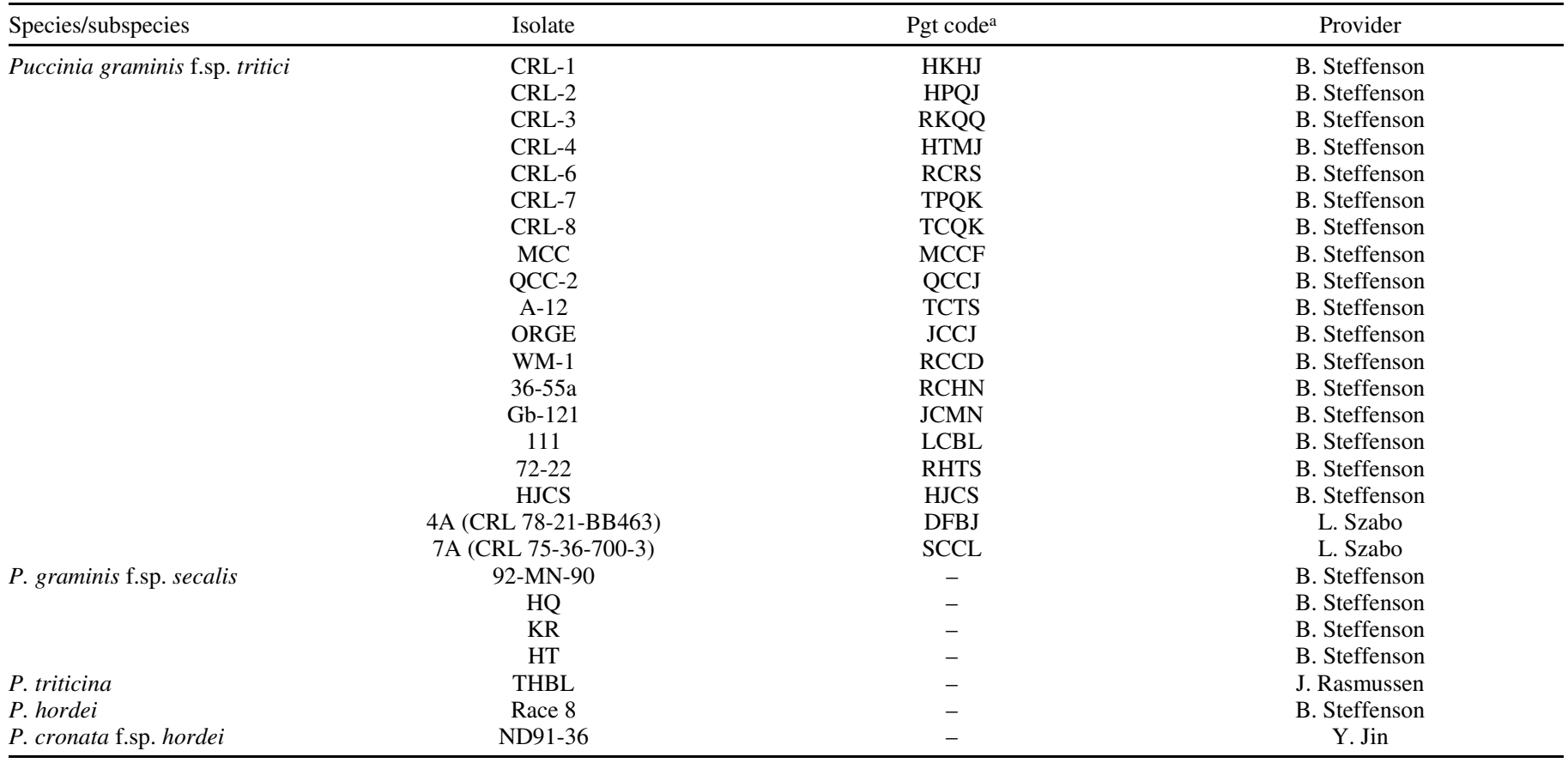

a The Pgt code is based on the reaction on a set of 16 stem rust differentials (27). The - indicates data were not applicable. 
transferability. Polymorphism information content (PIC) for each EST-SSR locus was calculated using the online program. All DNA samples used in the study were extracted from urediniospores using a FastDNA kit (MP Biomedicals, Solon, $\mathrm{OH}$ ) and a FastPrep Instrument (MP Biomedicals, Solon, $\mathrm{OH}$ ) according to the manufacturer's protocol.

Genetic diversity analysis. The alleles of each SSR locus amplified from individual isolates of $P$. graminis $\mathrm{f}$. $\mathrm{sp}$. tritici and $P$. graminis f. sp. secalis were scored and designated as 0 or 1 according to the absence or presence of the allele. Genetic similarity between isolates was measured as coefficients using the SimQual program of NTSYSpc version 2.021 (Exeter Software, Setaukal, NY). The unweighted pair group method of arithmetic averages (UPGMA) (SAHN program, NTSYSpc version 2.021) was used to construct a phenogram representing genetic similarities based on the SSR data. To estimate the strength of the groupings generated by the cluster analysis, bootstrap analysis was conducted with 500 replications using the Winboot computer program (25).

\section{RESULTS}

Identification of SSRs in EST sequences. The 60,579 EST sequences generated from three cDNA libraries of $P$. graminis $\mathrm{f}$. sp. tritici (37) were clustered into 11,853 unique sequences, which consisted of 4,184 contigs and 7,669 singletons. A database was constructed for these unique sequences and used to search for the 14 unique classes of di- and tri-nucleotide repeats described by Jurka and Pethiyagoda (16) and Katti et al. (19). A total of 708 unique sequences (contigs or singletons) were found to contain microsatellites with repeat numbers equal to or greater than 6 . The number of repeat units ranged from 6 to 14, but the majority (75\%) of the microsatellites had six or seven repeat units. All 14 unique classes of di- and trinucleotide repeats were found, but the frequencies of the classes varied among the 708 microsatellites (Fig. 1). Class 1 (AT/TA), 2 (AG/GA/CT/TC), and 3 (AC/CA/ TG/GT) were the most frequent of the dinucleotide classes, which had 68, 218, and 120 occurrences, respectively. Class 6 (AAG/ AGA/GAA/CTT/TTC/TCT), 7 (AAG/AGA/GAA/CTT/TTC/TCT), and 8 (ATG/TGA/GAT/CAT/ATC/TCA) were the most frequent trinucleotide classes, which had 50, 99, and 64 occurrences, respectively. The remaining classes were found from 3 to 21 times each (Fig. 1).
To map the microsatellite loci to the draft $P$. graminis $\mathrm{f}$. sp. tritici genome sequence, BLASTN searches were conducted against the 4,557 contigs in 392 scaffolds (supercontigs) assembled from the genome sequence reads of $P$. graminis $\mathrm{f}$. sp. tritici. All of the 708 microsatellite loci were mapped onto contigs based on their BLASTN matches at an E-value of $1 \times 10^{-25}$ or less. We selected 414 of the loci for further primer design based on their location and distribution on contigs and supercontigs of the $P$. graminis $\mathrm{f}$. sp. tritici genome. BLASTX searches against the GenBank database were conducted with these 414 sequences. However, most of them did not have matches or had similarity to sequences with no useful annotation (e.g., hypothetical or unnamed protein) in the database when an E-value of $1 \times 10^{-5}$ was used as cutoff (data not shown). Among the 72 microsatellite loci listed in Table 2, only seven had matches with meaningful annotation (data not shown).

Amplification and polymorphism. Primer pairs were successfully developed for 384 of the 414 microsatellite loci selected above. For the remaining 30 microsatellite loci, primer design was unsuccessful either because the repeats were too close to an end of the sequence or the flanking sequences did not meet the parameters set for primer design. The 384 primer pairs were initially screened for amplification on the DNA sample isolated from the isolate QCC-2. Only 202 of the primer pairs showed good amplification. These 202 primer pairs were further evaluated on 19 isolates of $P$. graminis f. sp. tritici and 4 isolates of $P$. graminis f. sp. secalis for polymorphism. Only 72 gave clear, easily scored, polymorphic banding patterns across at least 20 of the 23 isolates (Fig. 2). These 72 primer pairs were considered useful and were included in Table 2. The remaining 130 were not included in Table 2 because they either didn't show polymorphism, or gave amplification in some isolates, but not in others, or the bands amplified by them were too numerous to be resolved, or not of the expected size.

Among the 72 primer pairs shown in Table 2, 53 were polymorphic between the two isolates 4A (CRL 78-21-BB463) and 7A (CRL 75-36-700-3), which were used to make a mapping population for the development of a partial genetic map of $P$. graminis f. sp. tritici (36). An additional 46 primer pairs were also found to be polymorphic between isolates $4 \mathrm{~A}$ and $7 \mathrm{~A}$ when the remaining 130 primer pairs were evaluated (data not shown). This gave a total number of 99 primer pairs exhibiting polymorphism between these two parental isolates.

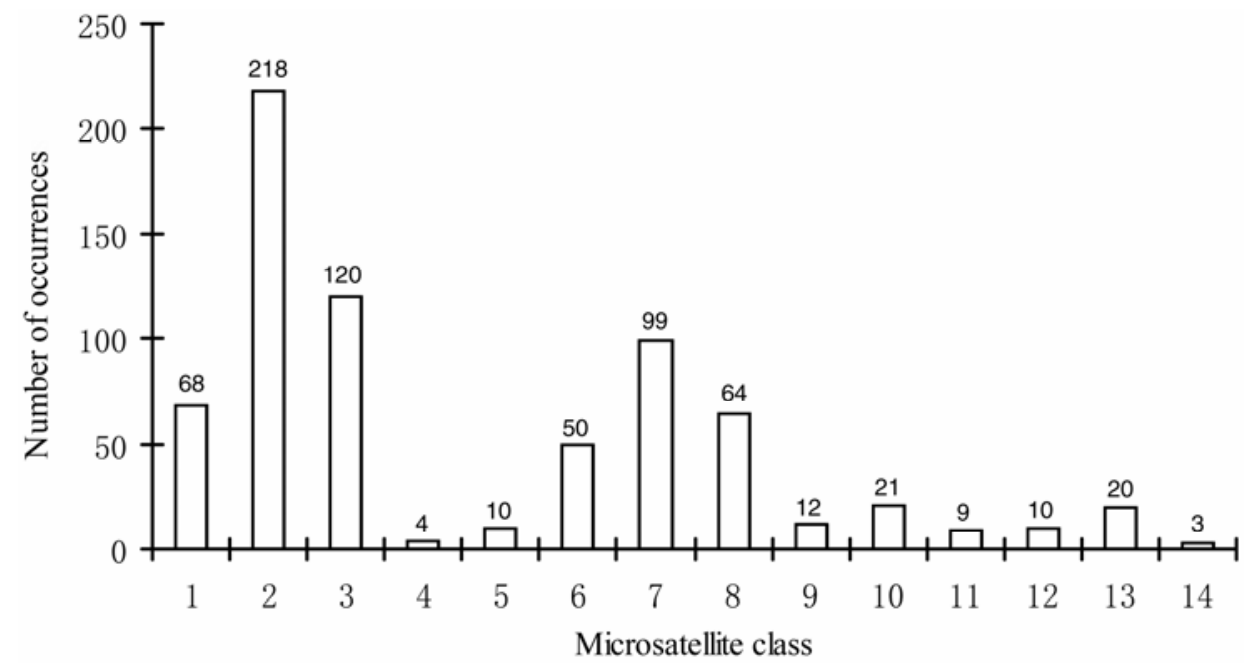

Fig. 1. Frequency distribution of the 14 unique classes of di- and tri-nucleotide repeats $(16,19)$ among 708 potential microsatellite loci identified from the expressed sequence tag database of Puccinia graminis f. sp. tritici. Microsatellite classes are as follows: class $1=\mathrm{AT} / \mathrm{TA}$; class $2=\mathrm{AG} / \mathrm{GA} / \mathrm{CT} / \mathrm{TC}$; class $3=$ $\mathrm{AC} / \mathrm{CA} / \mathrm{TG} / \mathrm{GT}$; class $4=\mathrm{GC} / \mathrm{CG}$; class $5=\mathrm{AAT} / \mathrm{ATA} / \mathrm{TAA} / \mathrm{ATT} / \mathrm{TTA} / \mathrm{TAT} ;$ class $6=\mathrm{AAG} / \mathrm{AGA} / \mathrm{GAA} / \mathrm{CTT} / \mathrm{TTC} / \mathrm{TCT}$; class $7=\mathrm{AAC} / \mathrm{ACA} / \mathrm{CAA} /$ GTT/TTG/TGT; class $8=$ ATG/TGA/GAT/CAT/ATC/TCA; class $9=$ AGT/GTA/TAG/ACT/CTA/TAC; class $10=$ AGG/GGA/GAG/CCT/CTC/TCC; class $11=$ AGC/GCA/CAG/GCT/CTG/TGC; class $12=$ ACG/CGA/GAC/CGT/GTC/TCG; class $13=$ ACC/CCA/CAC/GGT/GTG/TGG; class $14=$ GGC/GCG/CGG/ $\mathrm{GCC} / \mathrm{CCG} / \mathrm{CGC}$. 
Genetic diversity. A total of 359 polymorphic alleles were generated from the 72 SSR loci across 19 isolates of $P$. graminis $\mathrm{f}$. sp. tritici and 4 isolates of $P$. graminis $\mathrm{f}$. sp. secalis. The number of alleles ranged from 2 to 10, with an average of 5 alleles per locus. The presence or absence of an allele in each isolate was scored as 1 or 0 , respectively. Distance metrics were calculated, and a UPGMA dendrogram was derived to assess genetic diversity among the 23 isolates of $P$. graminis (Fig. 3). The resulting phenetic dendrogram showed that 22 of the 23 samples had distinct genotypes. Isolates CRL-2 and CRL-4 had the same genotypes based on the SSR loci evaluated. However, these two isolates showed different virulence phenotypes (Table 1); they could be derived from the same lineage but with mutations at the virulence loci (5). Two (92-MN-90 and HQ) of the four $P$. graminis f. sp. secalis isolates formed a cluster, which were separated from other $P$. graminis f. sp. tritici isolates. The other two rye stem rust isolates (KR and HT) were clustered with several other $P$. graminis f. sp. tritici isolates (CRL-2, -3, -4, -7, and -8 ) and appeared to be more closely related to $P$. graminis $\mathrm{f}$. sp. tritici than to $P$. graminis f. sp. secalis. Other small clusters were also identified, for example, CRL-1 and HJCS, 36-55a and 4A, 72-22 and CRL-6, and A-12. These groupings were supported by the bootstrapping analysis with a bootstrap value $>70$ (Fig. 3 ).

Cross-species amplification. To test the transferability of the EST-SSR primers developed from ESTs of $P$. graminis f. sp. tritici to other rust species, 32 of the 72 primer pairs were used for PCR with DNA templates from one isolate of P. triticina, P. hordei, and $P$. cronata f. sp. hordei, respectively. The results showed that nine of the 32 primer pairs produced amplicons in the $P$. triticina isolate THBL and nine primer pairs in race 8 of $P$. hordei (Table 2 ). Seven of the primer pairs also amplified DNA fragments from the barley crown rust isolate ND91-36 (Table 2).

\section{DISCUSSION}

Our results indicated that the EST sequences generated from $P$. graminis f. sp. tritici provided a rich source of additional microsatellite markers for population genetics studies in the fungus. Among the 11,853 unique sequences (including contigs and singletons) assembled from a total of 60,579 EST sequences, approximately $6 \%(708 / 11853)$ contained microsatellites. The discovery rate is higher than that reported for other fungi, which have di- or tri-nucleotide microsatellites in 1.3 to $4.4 \%$ of the EST sequences (11). The reason for the high number of microsatellitecontaining ESTs in $P$. graminis f. sp. tritici is not known. $P$. graminis f. sp. tritici has a larger genome size compared to most other fungi that have been sequenced, but this is probably not the major reason for the high occurrence of SSRs in the EST database because the genome size is neither inversely nor directly proportional to the SSR abundance as has been reported for other genomes (18). It is possible that the dikaryotic nature of the fungus may contribute to the high number of SSRs in the EST sequences because the expansion of SSRs is more tolerated in diploid organisms than in haploid fungi (35). Lim et al. (23) compared the abundance of microsatellites in 14 fungal genomes and also found that Candida albicans, the only diploid fungus included in the study, possesses higher number of mono- to hexanucleotides than any other fungal genomes analyzed.

Among the 708 microsatellites, 410 were dinucleotide and 398 were trinucleotide repeats. The result is in contrast to those that have been reported previously in EST databases from other organisms $(17,33)$ where trinucleotide microsatellites are more abundant. The most commonly identified individual microsatellite in the $P$. graminis f. sp. tritici EST database was the dinucleotide $(\mathrm{AG})_{\mathrm{n}}$, followed by $(\mathrm{AC})_{\mathrm{n}}$. A high frequency of $(\mathrm{AG})_{\mathrm{n}}$ and $(\mathrm{AC})_{\mathrm{n}}$ microsatellites was also reported for plants $(17,33)$ and other fungi $(11,18,23)$. The $(\mathrm{AT})_{\mathrm{n}}$ microsatellite class was the first- or second-most frequent in most other fungi $(18,23)$ while they were the third-most frequent class in our $P$. graminis f. sp. tritici ESTs. However, the frequency of $(\mathrm{AT})_{\mathrm{n}}$ microsatellites in the $M$. graminicola EST database was low (11). For the trinucleotide microsatellites, differences in frequency were also observed between the $P$. graminis f. sp. tritici database and those for other fungi. For example, $(\mathrm{AAC})_{\mathrm{n}}$ was the most common trinucleotide repeat in the $P$. graminis f. sp. tritici database, but occurs less common in several other fungal genomes $(18,23)$. $(\mathrm{CCG})_{\mathrm{n}}$ was the least common microsatellite in the ESTs of $P$. graminis $\mathrm{f}$. sp. tritici and was the case for most other fungal genomes $(18,23)$, but it was the most frequent in Magnaporthe grisea (18) and moderately common in M. graminicola (11). Also, $(\mathrm{CCG})_{\mathrm{n}}$ microsatellites were the most common in several plant species, including barley (33) and sugarcane (9). All these results indicate that the relative abundance of each class of SSRs varies with different organisms although some similarity exists, as has been suggested by Lim et al. (23) and Karaoglu et al. (18).

We initially evaluated 384 primer pairs for PCR amplification with the DNA isolated from the isolate QCC-2, but failed to generate amplicons with 182 of the primer pairs after at least two attempts. There were several possible reasons for the failure of PCR amplification from the EST-SSR loci. First, for simplicity and convenience, we used the same annealing temperature $\left(52^{\circ} \mathrm{C}\right)$ in PCR for all the primers. This might be suitable for some primers but not for others. Optimization of PCR conditions may improve amplification efficiency. Second, the primers were designed based on EST sequences. Introns or other structures in the genome may have prevented amplification. When the introns were too long or the primers were designed from the adjoining regions of an intron, no amplification would occur. Comparison of the EST-SSR loci with the sequenced genome sequence indicated that some of the failed primers flanked a large intron $(>1.5 \mathrm{~kb})$ or mapped to the region where there was no sequence match (data not shown). Dracatos et al. (10) provided another evidence of the presence of introns in a number of EST-SSR loci of the crown rust fungus ( $P$. coronata $\mathrm{f}$. sp. lolli) by sequence analysis. Third, since there was heterogeneity between the dikaryotic nuclei of the urediniospore and the ESTs were generated from the isolate 7A used for the whole genome sequencing, point mutations, or insertions and deletions within the priming sites could also account for PCR failure in other isolate (i.e., QCC-2). We aligned the primer sequences to the genomic sequence of isolate 7A and found the majority of the failed primer (s) showed 1 to $4 \mathrm{bp}$ mismatch to the genomic DNA (data not shown).
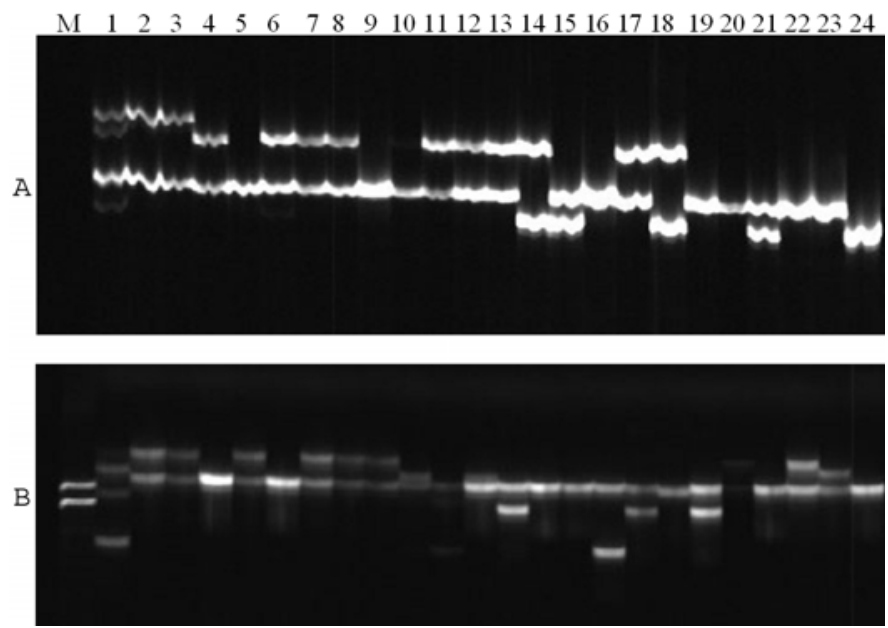

Fig. 2. Polymorphism revealed by the expressed sequence tag derived microsatellite markers, Pgestssr161 (A) and Pgestssr269 (B), among four isolates of Puccinia graminis f. sp. secalis and 20 isolates of $P$. graminis f. sp. tritici. M. 100-bp DNA ladder. No. 1, 10, 11, and 12 are P. graminis f. sp. secalis isolates and the others are $P$. graminis $\mathrm{f}$. $\mathrm{sp}$. tritici isolates. 


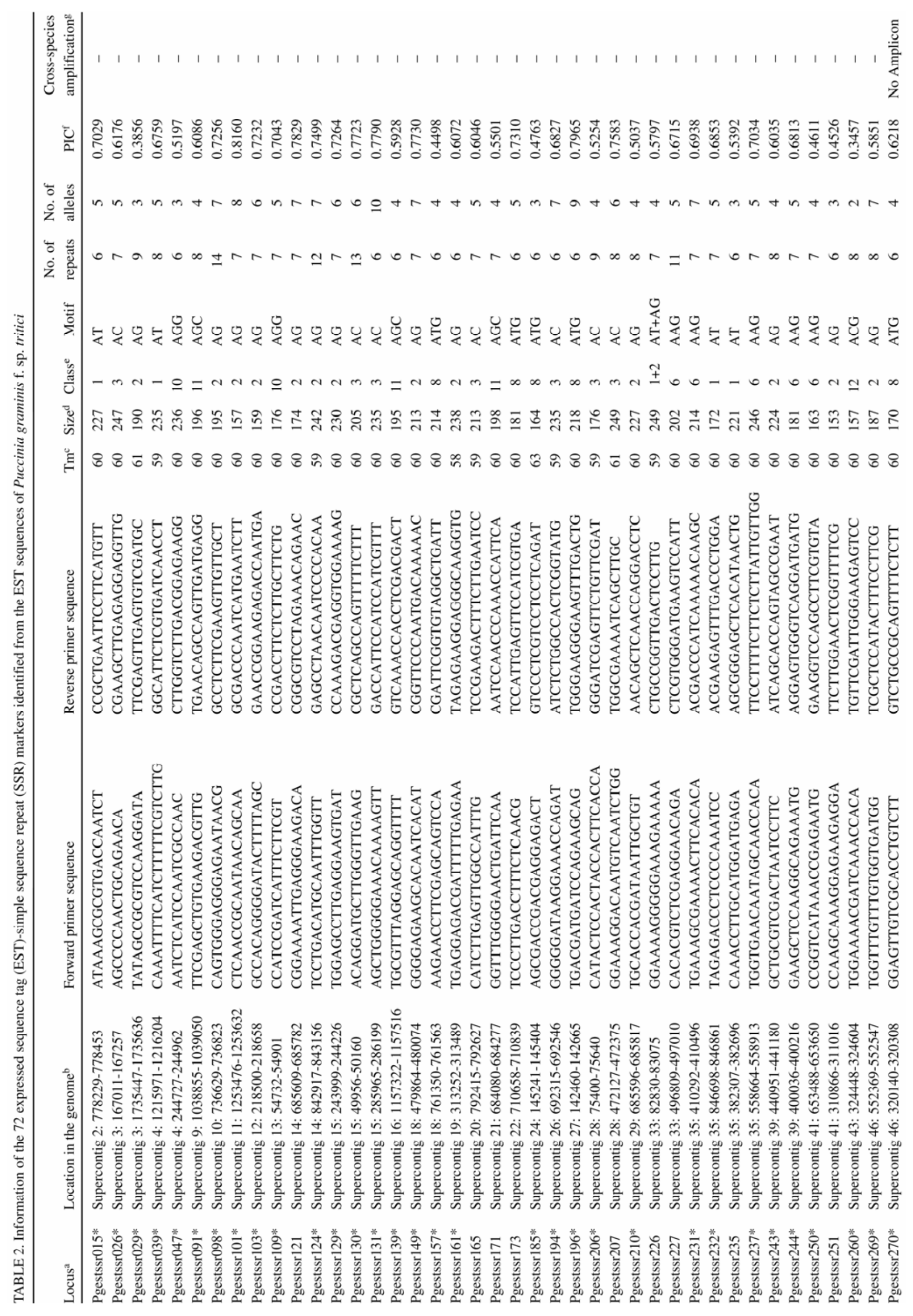




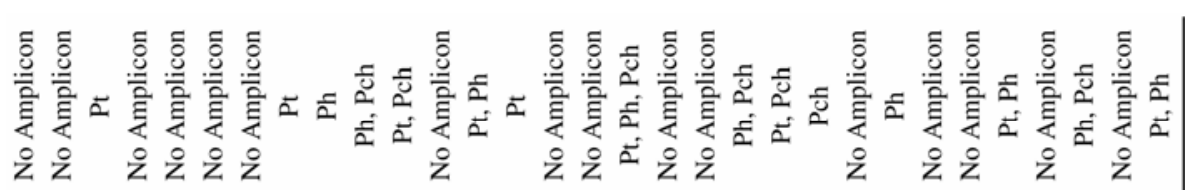

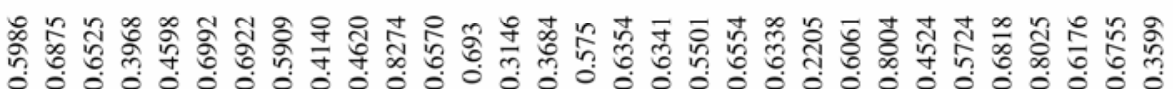

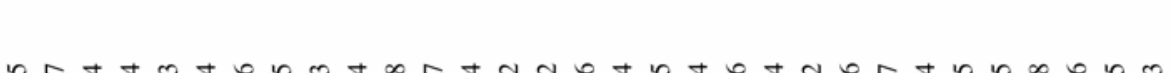

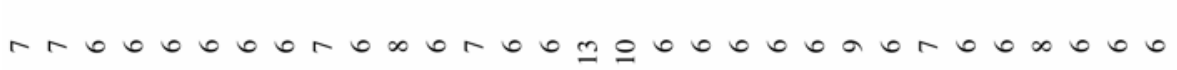

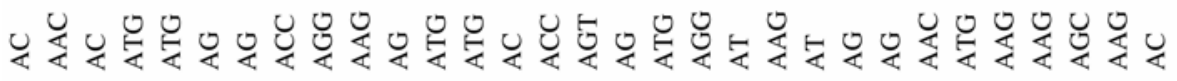

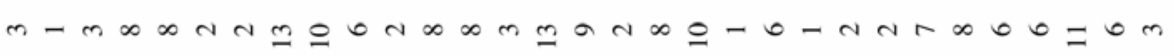
สิ

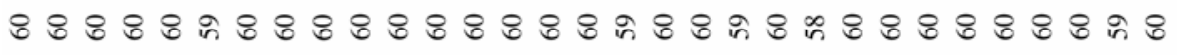

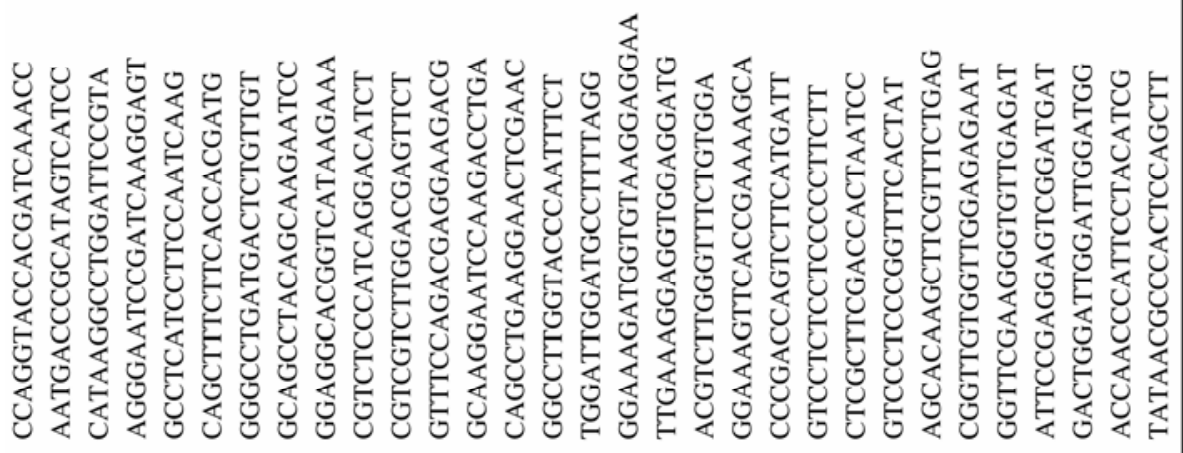

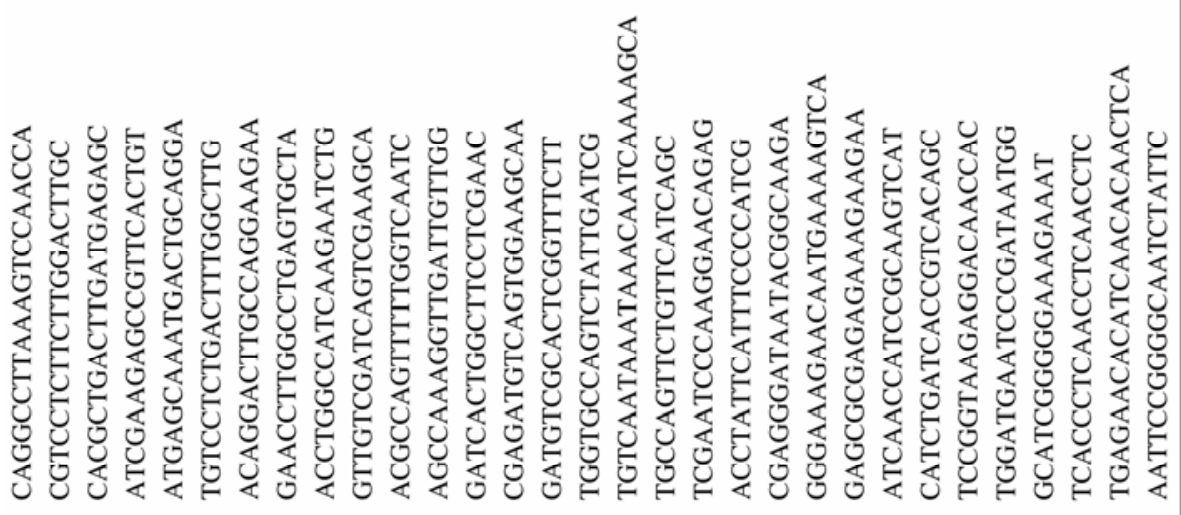

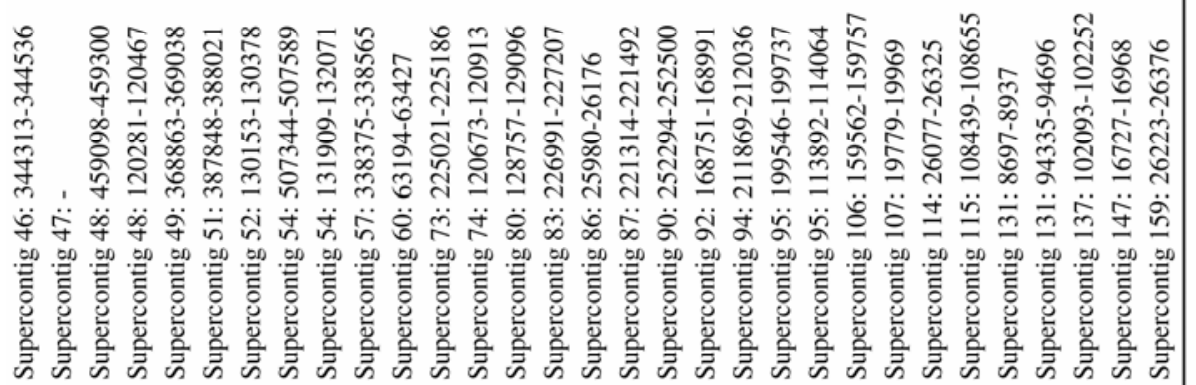

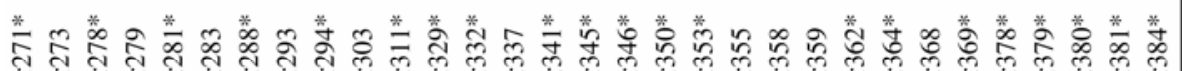

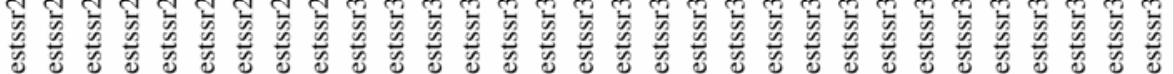

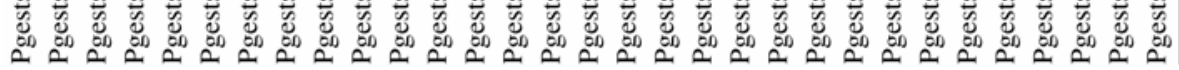

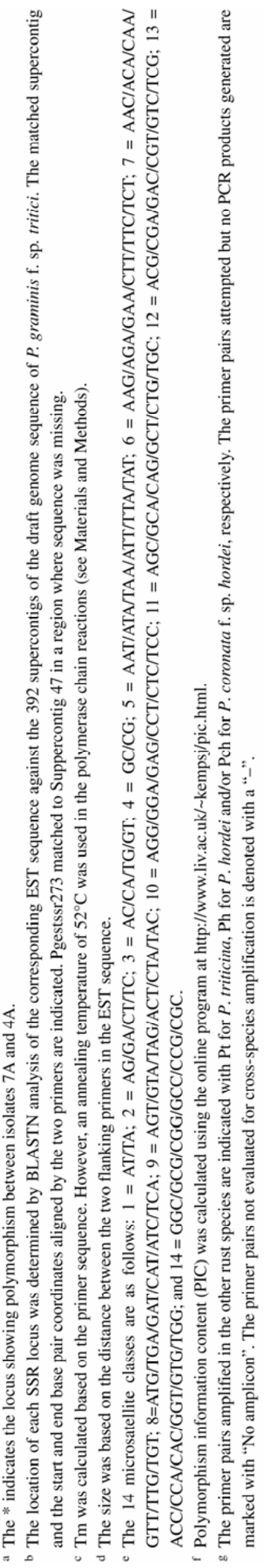


Therefore, even if the preliminary screening of the primers were done with the DNA of isolate 7A as template, many of the primers would still not amplify. The low efficiency and specificity in PCR amplification for EST-SSR loci were also observed by Dracatos et al. (10) who showed only 12 of 55 primer pairs evaluated in $P$. coronata $\mathrm{f}$. sp. lolli successfully amplified DNA fragments in this species. It appeared that the success rate of efficient primers for rust fungi with dikaryotic nuclei is lower compared to the haploid ascomycete fungi. This is probably due to the fact that rust fungi generally have larger genome sizes and more complex genome structures, such as a higher frequency of repetitive DNA sequences and higher DNA polymorphism within a single cell.

With the draft genome sequence available, we were able to localize the 72 SSR loci in 72 different contigs and 53 of the 392 supercontigs assembled from the genome sequences of $P$. graminis f. sp. tritici. Knowledge of their locations in the genome is important for population genetic studies because if markers clustered in the same region were selected for a genetic diversity analysis, the measurement of gametic disequilibrium might be inappropriate or biased. Using a mapping population derived from the cross between isolates $4 \mathrm{~A}$ and 7A, a partial genetic linkage map was developed, which consisted of mostly AFLP and RAPD markers along with avirulence loci (36). The 99 SSR markers polymorphic between the two parental isolates (4A and 7A) provide additional markers for the genetic linkage map. The saturation of the genetic map with these EST-SSR loci will not only assist in map-based gene cloning but also facilitate the assembly of the whole genome sequence. For example, if EST-SSR loci from different supercontigs are found linked to the same linkage group, the supercontigs can be assigned to the same chromosome.

Twenty-five SSR markers were developed from the SSRenriched genomic DNA libraries of $P$. graminis $\mathrm{f}$. $\mathrm{sp}$. tritici, but none of them were amplified in other rust fungi evaluated (32). Our study showed that many of the EST-SSR primers from $P$. graminis f. sp. tritici generated amplicons from three other rust species tested, including $P$. hordei, $P$. triticina, and $P$. coronata $\mathrm{f}$. sp. hordei. The high transferability of EST-SSRs has been observed in other fungal species. For example, Goodwin et al. (11) tested 99 primer pairs designed from the M. graminicola EST database on the closely related species Septoria passerinii and found that $66 \%$ of them amplified. Eight of twelve primer pairs tested also amplified on the more distantly related species $M$. fijiensis (11). Dracatos et al. (10) used 55 primer pairs for ESTSSR loci of $P$. coronata $\mathrm{f}$. sp. lolii to amplify the DNA from various fungal ( $P$. cronata f. sp. avenae, $P$. striiformis f. sp. tritici, Neotyphodium lolii, Blumeria graminis, Aspergillus nidulans, and Penicillium marneffei) and plant (Lolium perenne) species and had a success rate of amplification ranging from 22 to 53\%. This suggests that SSR primers developed from EST sequences may be more easily transferred to other related species. The more closely related the organisms, the higher the rate of the transferability due to more closely related species sharing more homology in SSRcontaining genes. High transferability of EST-SSR markers is one of the advantages over the method of generating SSR markers derived from non-coding genomic regions.

Our results indicate that $P$. graminis $\mathrm{f}$. sp. secalis is very closely related to $P$. graminis $\mathrm{f}$. $\mathrm{sp}$. tritici since most of the 72 primer pairs amplified in the four $P$. graminis f. sp. secalis isolates. Interestingly, the four $P$. graminis f. sp. secalis isolates didn't form a distinct cluster different from the $P$. graminis f. sp. tritici isolates based on the 72 EST-SSR loci. Two of the rye stem rust isolates were indistinguishable from several of the wheat stem rust isolates (Fig. 3). This result further confirms those of Burdon and Marshall (3) and Anderson and Pryor (2) who demonstrated that these two formae speciales were very closely related to each other. Burdon et al. (4) also showed that some virulent isolates could be derived from somatic hybridization of these two formae speciales and were confirmed using isozymic and RFLP markers.

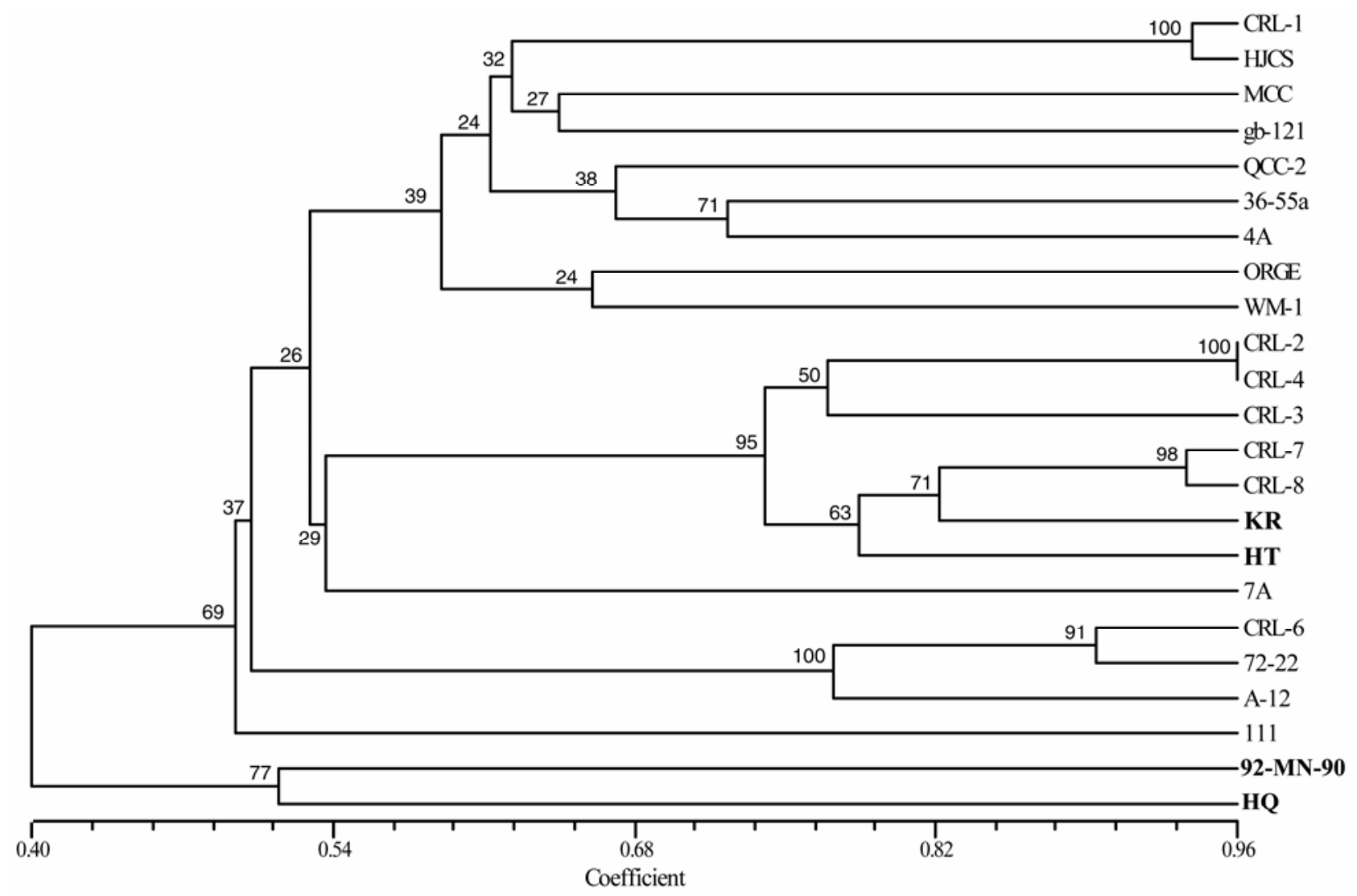

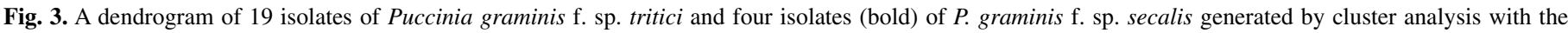

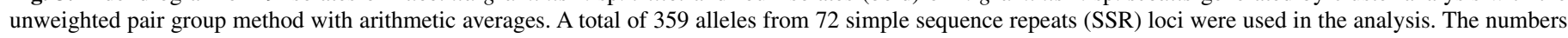
at the nodes of major clusters represent bootstrap values generated by 500 replications using the Winboot program (25). 
The two rye stem rust isolates KR and HT were not only virulent on rye plants but also infected most of the wheat stem rust differentials (unpublished data). Therefore, further investigation is needed to reveal the relationship of these rye stem rust isolates with the wheat stem rust isolates.

Roelfs et al. (28) showed that the American isolates could be separated into eleven clusters based on similarity in isozymic and virulence loci. In general, our cluster analysis of isolates was in agreement with those of Roelfs et al. (28). For example, QCC-2, a race that attacked barley with the resistance gene Rpg1, was separated from other North American isolates. Several other clusters in our study correspond to those of Roelfs et al. (28), including CRL-8 (T__K), CRL-3 (R__Q), CRL-1 (Q_CQ), MCC (MCC_), CRL-6 (RCRS), and 72-22 (RHTS). It is notable that CRL-6 (RCRS) and 72-22 (RHTS) are very closely related and probably should be considered the same cluster. These two isolates were also very similar in the analyses of Burdon and Roelf (7) and Roelfs et al. (28) although they were separated into two different clusters by these authors. The isolates $4 \mathrm{~A}$ and $7 \mathrm{~A}$ were from the sexually reproducing North American population of $P$. graminis $\mathrm{f}$. sp. tritici (36); they were separated from other isolates derived from asexually reproducing populations except for isolate 36-55a which showed some relationship with isolate 4A. Isolate 36-55a was collected in 1936 (A. Roelfs, personal communication) and was probably derived from a sexually reproducing population due to the barberry plants not being completely eradicated at that time.

\section{ACKNOWLEDGMENTS}

We thank B. Yang for DNA extraction and assistance in PCR. We acknowledge Z. J. Tu for providing the Perl scripts for sequence analysis. We also thank J. Rasmussen at North Dakota State University, B. Steffenson at University of Minnesota, and Y. Jin at USDA-ARS Cereal Disease Lab for providing rust samples used for this research. The Broad Institute provided the genomic sequence of Puccinia graminis through the Microbial Genome Sequencing Program funded by the National Science Foundation.

\section{LITERATURE CITED}

1. Altschul, S. F., Madden, T. L., Schaffer, A. A., Zhang, J., Zhang, Z., Miller, W., and Lipman, D. J. 1997. Gapped BLAST and PSI-BLAST: A new generation of protein database search programs. Nucleic Acids Res. 25:3389-3402.

2. Anderson, P. A., and Pryor, A. J. 1992. DNA restriction fragment length polymorphisms in the wheat stem rust fungus, Puccinia graminis $\mathrm{f}$. sp. tritici. Theor. Appl. Genet. 83:715-719.

3. Burdon, J. J., and Marshall, D. R. 1981. Isozyme variation between species and formae speciales of the genus Puccinia. Can. J. Bot. 59:2628-2634.

4. Burdon, J. J., Marshall, D. R., and Luig, N. H. 1981. Isozyme analysis indicates that a virulent cereal rust pathogen is a somatic hybrid. Nature 293:3565-3566.

5. Burdon, J. J., Marshall, D. R., Luig, N. H., and Gow, D. J. S. 1982. Isozyme studies on the origin and evolution of Puccinia graminis $\mathrm{f}$. $\mathrm{sp}$. tritici in Australia. Aust. J. Biol. Sci. 35:231-238.

6. Burdon, J. J., and Roelfs, A. P. 1985. Isozyme and virulence variation in asexually reproducing populations of Puccinia graminis and $P$. recondita on wheat. Phytopathology 75:907-913.

7. Burdon, J. J., and Roelfs, A. P. 1985. The effect of sexual and asexual reproduction on the isozyme structure of populations of Pucinia graminis. Phytopathology 75:1068-1073.

8. Chen, X., Line, R. F., and Leung, H. 1995. Virulence and polymorphic DNA relationships of Puccinia striiformis f. $\mathrm{sp}$. hordei to other rusts. Phytopathology 85:1335-1342.

9. Cordeiro, G. M., Casu, R., McIntyre, C. L., Manners, J. M., and Henry, R. J. 2001. Microsatellite markers from sugarcane (Saccharum spp.) ESTs cross transferable to erianthus and sorghum. Plant Sci. 160:1115-1123.

10. Dracatos, P. M., Dumsday, J. L., Olle, R. S., Corgan, N. O. I., Dobrowolski, M. P., Fujimori, M., Roderick, H., Stewart, A. V., Smith, K. F., and Forster, J. W. 2006. Development and characterization of EST-SSR markers for the crown rust fungus (Puccinia coronata f. sp. lolli). Genome 49:571-583.

11. Goodwin, S. B., van der Lee, T. A. J., Cavaletto, J. R., Hekkert, B. L. Crane, C. F., and Kema, G. H. J. 2007. Identification and genetic mapping of highly polymorphic microsatellite loci from an EST database of the Septoria tritici blotch pathogen Mycosphaerella graminicola. Fungal Genet. Biol. 44:398-414.

12. Huang, X., and Madan, A. 1999. CAP3: A DNA sequence assembly program. Genome Res. 9:868-877.

13. Jin, Y., Pretorius, Z. A., Singh, R. P., and Fetch, T. 2008. Detection of virulence to resistance gene $\mathrm{Sr} 24$ within race TTKS of Puccinia graminis f. sp. tritici. Plant Dis. 92:923-926.

14. Jin, Y., and Singh, R. P. 2006. Resistance in U.S. wheat to recent eastern African isolates of Puccinia graminis f. sp. tritici with virulence to resistance gene Sr31. Plant Dis. 90:476-480.

15. Jin, Y., Singh, R. P., Ward, R. W., Wanyera, R., Kinyua, M. G., Njau, P., Fetch, T., Pretorius, Z. A., and Yahyaoui, A. 2007. Characterization of seedling infection types and adult plant infection responses of monogenic $\mathrm{Sr}$ gene lines to race TTKS of Puccinia graminis f. sp. tritici. Plant Dis. 91:1096-1099.

16. Jurka, J., and Pethiyagoda, C. 1995. Simple repetitive DNA sequences from primates: Compilation and analysis. J. Mol. Evol. 40:120-126.

17. Kantety, R. V., La Rota, M., Matthews, D. E., and Sorrells, M. E. 2002. Data mining for simple sequence repeats in expressed sequence tags from barley, maize, rice, sorghum and wheat. Plant Mol. Biol. 48:501-510.

18. Karaoglu, H., Lee, C. M. Y., and Meyer, W. 2005. Survey of simple sequence repeats in completed fungal genomes. Mol. Biol. Evol. 22:639-649.

19. Katti, M. V., Ranjekar, P. K., and Gupta, V. S. 2001. Differential distribution of simple sequence repeats in Eukaryotic genome sequences. Mol. Biol. Evol. 18:1161-1167.

20. Kolmer, J. A. 2001. Molecular polymorphism and virulence phenotypes of the wheat leaf rust fungus Puccinia triticina in Canada. Can. J. Bot. 79:917-926

21. Leonard, K. J. 2001. Black stem rust biology and threat to wheat growers. [Published Online]. Available at www.ars.usda.gov/Main/docs.htm?docid= $10755 \& \mathrm{pf}=1 \& \mathrm{cg} \_\mathrm{id}=0$. Cereal Disease Lab., St. Paul, MN.

22. Leonard, K. J., and Szabo, L. J. 2005. Stem rust of small grains and grasses caused by Puccinia graminis. Mol. Plant Pathol. 6:99-111.

23. Lim, S., Notley-McRobb, L., Lim, M., and Carter, D. A. 2004. A comparison of the nature and abundance of microsatellites in 14 fungal genomes. Fungal Genet. Biol. 41:1025-1036.

24. McCallum, B. D., Roefls, A. P., Szabo, L. J., and Groth, J. V. 1999. Comparison of Puccinia graminis f. sp. tritici from South America and Europe. Plant Pathol. 48:574-581.

25. Nelson, R. J., Baraoidan, M. R., Vera Cruz, C. M., Yap, I. V., Leach, J. E., Mew, T. W., and Leung, H. 1994. Relationship between phylogeny and pathotype for the bacterial blight pathogen of rice. Appl. Environ. Microbiol. 60:3275-3283.

26. Pretorius, Z. A., Singh, R. P., Wagoire, W. W., and Payne, T. S. 2000. Detection of virulence to wheat stem rust resistance gene Sr31 in Puccinia graminis f. sp. tritici in Uganda. Plant Dis. 84:203.

27. Roelfs, A. P., and Martens, J. W. 1988. An international system of nomenclature for Puccinia graminis f. sp. tritici. Phytopathology 78:526-533.

28. Roelfs, A. P., McCallum, B., McVey, D. V., and Groth, J. V. 1997. Comparison of virulence and isozyme phenotypes of Pgt-QCCJ and Great Plains races of Puccinia graminis f. sp. tritici. Phytopathology 87:910-914.

29. Rowell, J. B. 1984. Controlled infection by Puccinia graminis f. sp.tritici under artificial conditions. Pages 291-332 in: The Cereal Rusts, Vol. I. A. P. Roelfs and W. R. Bushnell, eds. Academic Press, New York.

30. Selkoe, K. A., and Toonen, R. J. 2006. Microsatellites for ecologists: A practical guide to using and evaluating microsatellite markers. Ecol. Lett. 9:615-629.

31. Sun, Y. L., and Steffenson, B. J. 2005. Reaction of barley seedlings with different stem rust resistance genes to Puccinia graminis $\mathrm{f}$. $\mathrm{sp}$. tritici and Puccinia graminis f. sp. secalis. Can. J. Plant Pathol. 27:80-89.

32. Szabo, L. J. 2007. Development of simple sequence repeat markers for the plant pathogenic rust fungus, Puccinia graminis. Mol. Ecol. Notes 7:92-94.

33. Thiel, W., Michalek, W., Varshney, R. K., and Graner, A. 2003. Exploiting EST databases for the development and characterization of gene-derived SSR markers in barley (Hordeum vulgare L.). Theor. Appl. Genet. 106:411-422.

34. Wanyera, R., Kinyua, M. G., Jin, Y., and Singh, R. 2006. The spread of stem rust caused by Puccinia graminis f. sp. tritici, with virulence on Sr31 in wheat in Eastern Africa. Plant Dis. 90:113.

35. Young, E. T., Sloan, J. S., and Van Riper, K. 2000. Trinucleotide repeats are clustered in regulatory genes in Saccharomyces cerevisiae. Genetics 154:1053-1068.

36. Zambino, P. J., Kubelik, A. R., and Szabo, L. J. 2000. Gene action and linkage of avirulence genes to DNA markers in the rust fungus Puccinia graminis. Phytopathology 90:819-826.

37. Zhong, S., Szabo, L., Jeong, J., Mitchell, T., Dean, R., and Cuomo, C. 2005. Development of full-length cDNA libraries for EST analysis of Puccinia graminis f. sp. tritici. (Abstr.) Phytopathology 95(suppl.):S118. 